\title{
The facilitative role of imagery in episodic memory: Multiple cues or active construction?
}

\author{
WILLIAM BEVAN and JAMES N. FEUER \\ Duke University, Durham, North Carolina 27706
}

\begin{abstract}
The purpose of this experiment was to compare free recall for pictorial and for image-mediated verbal stimuli. Five independent groups were tested. One received a set of 30 noun-verb-noun sentences and instructions for rote rehearsal; one received the same 30 trigrams and instructions to construct visual images; a third was asked simply to look at drawings portraying incidents suggested by the trigrams; the fourth group received Items 1-15 in the form of trigrams and Items 16-30 as pictures to be passively observed; for the fifth group, the trigram-picture sequence was reversed. Stimuli were presented only once. Presentation was followed by a 5-min distraction task, and then all subjects were asked to write down as many as they could remember of the names of the objects of the events depicted by the pictures and sentences (the third word in each trigram). There is some indication in the results that even passively observed pictures were recalled better-if only slightly better-than image-mediated words. This suggests that the multiple cues available in pictures are a better aid to recall than the processes of controlled imagery. However, a more important implication, drawn from the performance of the fourth group, is that a better approach to facilitating recall is to combine the advantages of each into a single strategy and to elaborate images from pictorial stimuli.
\end{abstract}

The psychological literature on imagery has had two predominant themes. The longer tradition has focused on autonomous imagery and has concerned itself, for the most part, with the phenomenal properties of images and their relation to internal states, primarily motivational and constitutional. The more recent revival of interest in imagery on the part of experimental psychologists has concentrated on controlled imagery as a cognitive vehicle in thinking and in the mediation of recall. The prevailing view excludes motivation as a determinant of the latter functions, on the grounds that the effectiveness of mnemonic devices are hard to conceive of in motivational terms (Paivio, 1968), that the possibility that imagery functions to enhance motivation through making the experimental task more interesting is difficult to demonstrate (Bower, 1972, p. 77), and that there are data on the differential effectiveness of imaginal and verbal mediators that a motivational hypothesis cannot accommodate (e.g., Bower, 1972, pp. 68-69; Pavio \& Foth, 1970). At the same time, there are also data that imply a motivational effect as a reasonable possibility (e.g., Sheehan, 1972; Smith \& Noble, 1965; Spiker, 1960). Furthermore, modern cognitive studies make it increasingly clear that both perception and memory involve active construction on the part of the person who perceives or remembers.

The prevailing hypothesis concerning the peculiar effectiveness of images in the facilitation of recall is framed in terms of the notion of multiple cues [cf. e.g.,

This experiment was part of a program of independent study conducted by J. N. Feuer under the direction of W. Bevan. the associative hooks discussion of Bevan, Dukes, and Avant (1966); the conceptual peg/dual-coding hypotheses of Paivio (1971, pp. 247-248, 233-242)]. The present paper reports an exploratory study aimed at differentiating between the cognitive mechanism associated with the multiple cues and the motivational effects derived from active construction as factors underlying the effectiveness of imaginal mediators in the free recall of episodes. It is based on the assumption that images derived from corresponding pictorial and verbal stimuli have the same properties as mediators. Consequently, the experimental strategy involved tests of free recall following the presentation of a series of pictures (P) portraying simple events (e.g., a cat smelling a flower), as well as after the presentation of a series of three-word sentences (S) describing the events (e.g., cat smell flower). Images associated with the pictures are assumed to be immediately given; those associated with the sentences are assumed to be actively constructed. If multiple cues are the essential mechanism, then recall of pictorial objects should be better than that of named objects; if active construction is the essential process, the opposite should be the case.

\section{METHOD}

\section{Experimental Design}

A total of 140 undergraduates, volunteers from introductory psychology courses, served as subjects. They were randomly assigned to five independent groups, with an equal number of male and female subjects in each. Two groups of 40 each constituted the groups of the basic experimental design, and the remaining three groups of 20 each were run to provide additional information on the effect of instructions and on possible sequence effects. The basic experiment involved a two-factor 
design with repeated measures (Winer, 1962, pp. 304-307), with the two main sources of variance being the type of stimuli presented to the subject (sentences vs. pictures) and order of presentation (sentences followed by pictures vs. pictures followed by sentences).

\section{Materials}

The stimulus sentences consisted of 30 different noun-verbnoun trigrams typed in capital letters on white bond, photographed, and made up as $2 \times 2$ in. slides. The nouns used in the trigrams were selected from the Paivio, Yuille, and Madigan (1968) list, from among those high on the concreteness and imagery scales, and were carefully organized into two matched sets of 15 trigrams each. Mean imagery values for the agents (subjects of the sentences) and objects (objects of the sentences) for Trigrams 1-15 were 6.52 (range: $6.10-6.87$ ) and 6.55 (range: 6.37-6.75), respectively; for Trigrams 16-30, 6.56 (range: 6.236.80) and 6.51 (range: 6.17-6.77), respectively. Mean concreteness values for the agents and objects of Trigrams 1-15 were 6.76 (range: $6.13-7.00$ ) and 6.89 (range: 6.12-7.00), respectively; for Trigrams 16-30, 6.85 (range: 6.20-7.00) and 6.92 (range: 6.73-7.00), respectively.

The picture stimuli were line drawings-black lines on a white field-depicting each of the 30 incidents indicated by the trigram sentences, also photographed and made up as $2 \times 2$ in. slides.

\section{Procedure}

Three forms of instruction were used. Subjects in one of the control groups designated to receive sentences throughout were told that they would be shown three-word sentences, each for $15 \mathrm{sec}$, and that they were to say each sentence to themselves over and over again until the next slide appeared, without thinking of its meaning. The other group receiving only sentences was told to take an episode suggested by the sentence and elaborate a visual image during the $15-\mathrm{sec}$ period. The respective roles of agent and object were explained, and two trigrams not in the list of 30 were used as examples, each on one practice trial, before presentation of the list. The control group receiving the pictures was told simply to sit back, relax, and look at the pictures as they were presented. After the 30th item had been presented, all groups were given a 5-min distraction task (crossing through the letter " $j$ " in a $71 / 2$ by 10 matrix of randomly ordered letters) followed by a 6-min free recall test, during which they were asked to write down the names of as many of the objects (the recipients of the action involved in an episode) as they could remember. Subjects in each group were tested in sets of as many as 8 or 10 , depending on when they could be scheduled.

The procedure with the mixed groups of the main design differed from that of the control only in that the stimuli were presented in sets of 15 with appropriate instructions before each set, and with the recall test consisting of two 3-min segments, one for pictures and one for object names. The order of stimulus presentation was the same for all five groups. In one of the mixed groups, Items 1-15 were sentences and Items $16-30$ were pictures; in the other, Items $1-15$ were pictures and Items 16-30 were sentences. (In order to check on the possible effect of breaking the presentation of the items into two 15-item sequences in contrast to one 30 -item sequence, two additional control groups were run that differed from the 30 -item sentence and the 30 -item picture group only in this regard. Performance levels were almost identical for the first and second halves of each group.)

In no case did the initial instructions to the subjects indicate that they would be asked to recall the stimuli they were to be shown. Furthermore, in order to maximize the influence of active construction, on the one hand, and multiple cues, on the other, and assuming that either pictures would be stored directly in pictorial form, or that images derived from them would be faithful pictorial reproductions, type of stimulus was deliberately confounded with type of instructions: passively observe pictures in contrast to actively elaborate images from sentences.

\section{RESULTS AND DISCUSSION}

\section{The Facilitating Effect of Imagery}

The results of the present experiment are indicated in Figure 1. The difference in the height of the two left-hand bars $[t(38)=3.76, p<.001]$ simply demonstrates the widely known fact that imagery facilitates recall and requires no further discussion here. Similarly, the difference between the first and third bars [ $\mathrm{t}(38)=$ $3.70, \mathrm{p}<.001$ ] confirms the already known superiority of pictures over words as stimuli (cf. Bevan \& Steger, 1971). Comparison of the picture with the word segments suggests recall of pictures to be slightly better, but this is not borne out across the board. The difference, for example, between the second and third bars is not significant $[\mathrm{t}(38)=.32, \mathrm{p}>.05]$. However, the failure to differentiate between pictures and visually mediated words as stimuli may be a reflection of the difference in instructions used with these two control

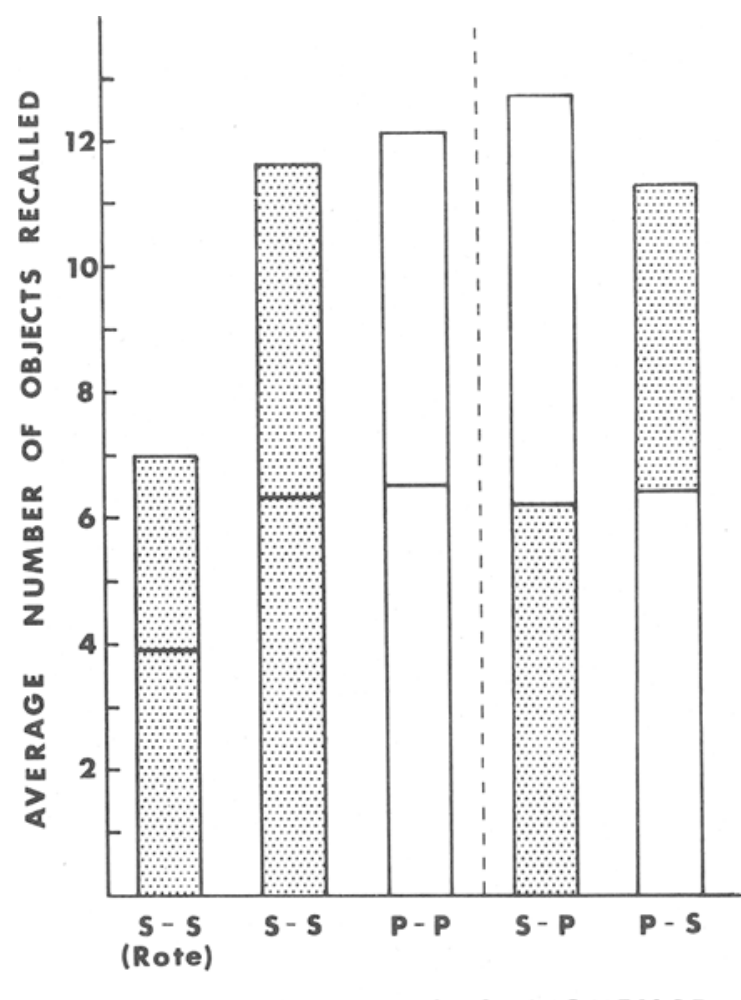

TYPE OF STIMULUS SEQUENCE

Figure 1. Average number of objects recalled by subjects in each of the five groups. Type of stimulus is indicated by the treatment on the bars: Clear white segments indicate recall of pictures; the stipled areas represent recall of object names. Recall of Items 1-15 is presented on the lower segment of each bar; for Items 16-30 on the upper segment. The letters $P$ and $S$ represent sentences and pictures, respectively. 
groups. That this may be the case is suggested by mixedgroup data to be described below.

\section{The Facilitation of Recall:}

\section{Multiple Cues or Active Construction?}

The two bars to the right of the dotted line in Figure 1 represent the performance of the mixed groups. Inspection suggests better performance with pictures than with mediated sentences. This was confirmed by statistical analysis [pictures vs. sentences $F(1,78)=9.09$, $\mathrm{p}<.01]$. Furthermore, this superiority proved to be a function of the prior influence of instructions to image upon the effectiveness of the stimulus properties of stimuli [stimulus type by order of presentation: $F(1,78)=5.50, p<.03]$. In contrast, the initial set to passively observe pictures would seem to adversely affect the effectiveness of image construction, for pictures followed by mediated sentences resulted in a $25 \%$ decrement in performance, a loss in the second segment of the presentation order relatively greater than that observed in the group receiving sentences throughout $(16 \%)$ or that in the rote learning group (19\%).

It is therefore impossible to conclude from the present results that the greater concreteness of pictures is a more potent determinant of free recall than the processes involved in the creation of an image. What can be suggested is that the influence of both of these variables can be shaped by the prior set of the subject. To understand the relation of pictures to images in recall, it is important to recognize that images are not mental pictures. Rather, looking at pictures and elaborating images are two strategies for encoding information that may be facilitated or interfered with as Atwood (1971) has shown. Indeed, the failure to demonstrate a marked difference in performance following pictures and following image-mediated sentences may reflect the fact that the recall may involve either (1) reviving images of equivalent quality from the memory store during the test and then translating them into verbal responses, or (2) constructing test responses from stored cues of equivalent quality.

\section{REFERENCES}

Atwood, G. An experimental study of visual imagination and memory. Cognitive Psychology, 1971, 2, 290-299.

Bevan, W., Dukes, W. F., \& Avant, L. L. The effect of variation in specific stimuli on memory for their superordinates. American Journal of Psychology, 1966, 79, $250-257$.

Bevan, W., \& Steger, J. A. Free recall and abstractness of stimuli. Science, 1971, 172, 597-599.

BoWER, G. H. Mental imagery and associative learning. In L. Gregg (Ed.), Cognition in learning and memory. New York: Wiley, 1972. Pp. 51-88.

Parvio, A. Effects of imagery instructions and concreteness of memory pegs in a mnemonic system. Proceedings of the 76th Annual Convention of the American Psychological Association, 1968. Pp. 68-69, 77.

PaIvio, A. Imagery and verbal processes. New York: Holt, Rinehart, \& Winston, 1971.

Paivio, A., \& Foth, D. Imaginal and verbal mediators and noun concreteness in paired-associate learning: The elusive interaction. Journal of Verbal Learning and Verbal Behavior, 1970, 9, 384-390.

Paivio, A., Yuille, J. C., \& Madigan, S. A. Concreteness, imagery, and meaningfulness values for 925 nouns. Journal of Experimental Psychology, 1968, 76(1, Part 2).

ShEEHAN, P. W. Role of imagery in incidental learning: Replication and extension of an effect. Journal of Experimental Psychology, 1972, 95, 226-228.

Smith, R. K., \& Noble, C. E. Effects of a mnemonic technique applied to verbal learning and memory. Perceptual and Motor Skills, 1965, 21, 123-134.

SPIKER, C. C. Associative transfer in verbal pairedassociate learning. Child Development, 1960, 31, 73-87.

WINER, B. J. Statistical principles in experimental design. New York: McGraw-Hill, 1962.

(Received for publication April 21, 1977.) 